\title{
Correction to: SERENA: Particle Instrument Suite for Determining the Sun-Mercury Interaction from BepiColombo
}

S. Orsini ${ }^{1}$ - S.A. Livi ${ }^{2,3}$ - H. Lichtenegger ${ }^{4}$ - S. Barabash ${ }^{5}$ - A. Milillo ${ }^{1}$ - E. De Angelis ${ }^{1}$.

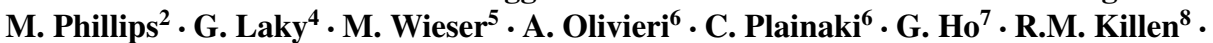
J.A. Slavin ${ }^{3}$ - P. Wurz' • J.-J. Berthelier ${ }^{10}$ • I. Dandouras ${ }^{11}$ • E. Kallio ${ }^{12}$.

S. McKenna-Lawlor ${ }^{13}$ - S. Szalai ${ }^{14}$ - K. Torkar $^{4}$ - O. Vaisberg ${ }^{15}$ - F. Allegrini ${ }^{2}$. I.A. Daglis ${ }^{16,17}$ - C. Dong ${ }^{18}$ - C.P. Escoubet ${ }^{19}$ - S. Fatemi ${ }^{5}$ - M. Fränz ${ }^{20}$ • S. Ivanovski ${ }^{21}$. N. Krupp ${ }^{20}$ - H. Lammer ${ }^{4}$ - François Leblanc ${ }^{10}$ - V. Mangano ${ }^{1}$ - A. Mura ${ }^{1}$ H. Nilsson ${ }^{5}$. J.M. Raines ${ }^{3}$ - R. Rispoli ${ }^{1}$ - M. Sarantos ${ }^{8}$ H.T. Smith ${ }^{7}$ K. Szego ${ }^{14}$ - A. Aronica ${ }^{1}$. F. Camozzi' 2 - A.M. Di Lellis ${ }^{23}$ - G. Fremuth ${ }^{4}$ F. Giner ${ }^{4}$ - R. Gurnee G $^{24}$ J. Hayes ${ }^{7}$. H. Jeszenszky ${ }^{4}$. F. Tominetti ${ }^{22}$ - B. Trantham ${ }^{2} \cdot$ J. Balaz $^{25}$ - W. Baumjohann ${ }^{4}$. D. Brienza ${ }^{1}$ - U. Bührke ${ }^{20}$ - M.D. Bush ${ }^{9}$ - M. Cantatore ${ }^{22}$ - S. Cibella ${ }^{26}$ - L. Colasanti ${ }^{1}$. G. Cremonese $^{27}$ - L. Cremonesi ${ }^{22}$ - M. D'Alessandro ${ }^{28}$ • D. Delcourt ${ }^{29}$ • M. Delva ${ }^{4}$. M. Desai ${ }^{2}$ - M. Fama $^{30}$ - M. Ferris ${ }^{2}$ H. Fischer ${ }^{20}$ - A. Gaggero ${ }^{26}$ - D. Gamborino' ${ }^{9}$ P. Garnier ${ }^{11}$ - W.C. Gibson ${ }^{2}$ - R. Goldstein ${ }^{2}$ - M. Grande ${ }^{31}$ - V. Grishin ${ }^{15}$ - D. Haggerty . $^{7}$ M. Holmström ${ }^{5}$ - I. Horvath ${ }^{14}$ - K.-C. Hsieh ${ }^{32}$ - A. Jacques ${ }^{8}$ - R.E. Johnson ${ }^{33}$. A. Kazakov ${ }^{1}$ - K. Kecskemety ${ }^{14}$ - H. Krüger ${ }^{20}$ - C. Kürbisch ${ }^{4}$. F. Lazzarotto ${ }^{27}$. Frederic Leblanc ${ }^{34}$ - M. Leichtfried ${ }^{4}$ - R. Leoni ${ }^{26}$ - A. Loose $^{20}$ - D. Maschietti $^{35}$. S. Massetti ${ }^{1}$ - F. Mattioli ${ }^{26}$ - G. Miller ${ }^{2}$ D. Moissenko ${ }^{15}$ - A. Morbidini ${ }^{1}$ - R. Noschese $^{1}$. F. Nuccilli ${ }^{1}$ - C. Nunez ${ }^{2}$ N. Paschalidis ${ }^{8}$ - S. Persyn ${ }^{2}$ - D. Piazza ${ }^{9}$ - M. Oja ${ }^{5}$ - J. Ryno Re $^{36}$. W. Schmidt ${ }^{36}$ - J.A. Scheer ${ }^{37}$ - A. Shestakov ${ }^{15}$ - S. Shuvalov ${ }^{15}$ - K. Seki ${ }^{38}$ - S. Selci ${ }^{26}$. K. Smith ${ }^{2}$ - R. Sordini ${ }^{1}$ - J. Svensson ${ }^{39}$ - L. Szalai ${ }^{14}$ - D. Toublanc ${ }^{11}$ - C. Urdiales ${ }^{2}$. A. Varsani ${ }^{4} \cdot$ N. Vertolli ${ }^{1}$ - R. Wallner ${ }^{4} \cdot$ P. Wahlstroem ${ }^{9} \cdot$ P. Wilson ${ }^{2} \cdot$ S. Zampieri ${ }^{1}$

W.C. Gibson is deceased.

The original article can be found online at https://doi.org/10.1007/s11214-020-00787-3

\section{S. Orsini}

1 Institute of Space Astrophysics and Planetology, INAF, via del Fosso del Cavaliere 100, 00133, Rome, Italy

2 Southwest Research Institute, San Antonio, TX, USA

3 Department of Climate and Space Sciences and Engineering, University of Michigan, Ann Arbor, MI, USA 


\section{Correction to: Space Sci. Rev. (2021) 217:11 https://doi.org/10.1007/s11214-020-00787-3}

The original online version of this article was revised because a number of authors had the wrong affiliation number next to their names.

4 Space Research Institute, Austrian Academy of Sciences, Graz, Austria

5 Swedish Institute of Space Physics, Kiruna, Sweden

6 Italian Space Agency, Roma, Italy

7 The Johns Hopkins University Applied Physics Laboratory, Laurel, MD 20723, USA

8 NASA/Goddard Space Flight Center, Greenbelt, MD 20771, USA

9 Physics Institute, University of Bern, Bern, Switzerland

10 LATMOS/IPSL, CNRS, Sorbonne Université, Paris, France

11 Institut de Recherche en Astrophysique et Planétologie, CNRS, CNES, Université de Toulouse, Toulouse, France

12 School of Electrical Engineering, Department of Electronics and Nanoengineering, Aalto University, Helsinki, Finland

13 Space Technology Ireland, Ltd., Maynooth, Co. Kildare, Ireland

14 Wigner Research Centre for Physics, Budapest, Hungary

15 IKI Space Research Institute, Moscow, Russia

16 Department of Physics, National and Kapodistrian University of Athens, Athens, Greece

17 Hellenic Space Center, Athens, Greece

18 Department of Astrophysical Sciences and Princeton Plasma Physics Laboratory, Princeton University, Princeton NJ, USA

19 ESA-ESTEC, Noordwijk, The Netherlands

20 Max-Planck-Institut für Sonnensystemforschung, MPS, 37077 Göttingen, Germany

21 Astronomical Observatory, INAF, Trieste, Italy 
Publisher's Note Springer Nature remains neutral with regard to jurisdictional claims in published maps and institutional affiliations.

22 OHB-Italia SpA, Milano, Italy

23 AMDL srl, Roma, Italy

24 Laboratory for Atmospheric and Space Physics, Boulder, CO, USA

25 Institute of Experimental Physics SAS, Slovak Academy of Sciences, 04001 Košice, Slovakia

26 Istituto Fotonica e Nanotecnologie, CNR-IFN, Roma, Italy

27 Astronomical Observatory, INAF, Padova, Italy

28 Istituto di Struttura della Materia (CNR-ISM), 00133 Roma, Italy

29 University of Orleans, Orleans, France

30 Comisión Nacional de Energía Atómica, cnea, Centro Atómico Bariloche, Bariloche, Argentina

31 Aberystwyth University, Aberystwyth, Ceredigion, SY23 3FL, UK

32 University of Arizona, Tucson, AZ, USA

33 University of Virginia, Charlottesville, VA 22904, USA

34 LPP, École polytechnique, 91128 Palaiseau Cedex, France

35 PRISMA srl., Roma, Italy

36 Finnish Meteorological Institute FMI, Helsinki, Finland

37 TOFWERK, Thun, Switzerland

38 Department of Earth and Planetary Science, Graduate School of Science, University of Tokyo, Tokyo, Japan

39 EISCAT, Kiruna, Sweden 